\title{
Wissenschaftliche Politikberatung durch Ressortforschungseinrichtungen
}

\author{
Herausforderungen, Merkmale und Zukunftsperspektiven
}

Andreas Süssmilch

\section{Grundlagen und Aufgaben der Ressortforschung}

Im deutschen Wissenschaftssystem liegt das Hauptaugenmerk in der Forschungspolitik vorwiegend auf der Forschung an Hochschulen, an den „vier Säulen“ des außeruniversitären Forschungssystems (Max Planck Gesellschaft, Fraunhofer Gesellschaft, Leibnitz Gemeinschaft und Helmholtz Gemeinschaft) sowie in der Wirtschaft. Weniger Beachtung findet hingegen die Ressortforschung. Unter Ressortforschung versteht man die Forschungs- und Entwicklungsaktivitäten der Bundes- und Landesministerien in der Bundesrepublik Deutschland. Dabei werden Themenschwerpunkte behandelt, die von den Ressorts dauernd bzw. über sehr lange Zeit bearbeitet werden müssen. Dies führe zur Gründung sogenannter dem Ministerium nachgeordneten Ressortforschungseinrichtungen. Die vorliegende Abhandlung beschäftigt sich vorwiegend mit der Ressortforschung des Bundes.

Die Möglichkeit der Bundesministerien, in ihren Geschäftsbereichen eigene Ressortforschungseinrichtungen zu unterhalten, ergibt sich aus Artikel 87 (3) des Grundgesetzes. Danach kann der Bund für Angelegenheiten, für die ihm die Gesetzgebung zusteht, Bundesoberbehörden und bundesunmittelbare Anstalten des öffentlichen Rechts einrichten. Die Gesetzgebungskompetenzen des Bundes erstrecken sich laut Artikel 73 und 74 GG u. a. auf Bereiche wie Maße/Gewichte/ Zeitbestimmung, die Sicherung der Ernährung, den Arbeitsschutz, das Siedlungs- und Wohnungswesen, den Bundesfernstraßenbau, die Binnenschifffahrt, Verteidigung, Archäologie, Strahlenschutz, internationale Politik oder Maßnahmen gegen gefährliche und übertragbare Krankheiten bei Menschen und Tieren. Darüber hinaus hat der Staat Aufgaben im Bereich der technischen Sicherheit in eigener Verantwortung übernommen. Es existieren mehr als 50 Institutionen mit einem jährlichen Finanzvolumen von rd. 1,4 Mrd. Euro (Stand 2008), davon 0,64 Mrd. Euro für Forschung und Entwicklung. Das entspricht 13,4\% der 2008 seitens des Bundes für die institutionelle Förderung der Forschung verausgabten Mittel. Nach Erhebungen des Wissenschaftsrates verfügten die Einrichtungen mit Ressortforschungsaufgaben im Jahr 2008 insgesamt über 18.043 institutionelle Stellen, darunter 5.449 für FuEPersonal (Wissenschaftsrat, 2010). Etwa 10\% des öffentlich beschäftigten wissenschaftlichen Personals arbeitet damit in einer Ressortforschungseinrichtung.

Ressortforschung als ein eigenständiger Typ angewandter Forschung ist durch folgende Besonderheiten gekennzeichnet: Sie greift Fragen von Politik, Wirtschaft und Gesellschaft auf und ist damit normalerweise problemorientiert und praxisnah. Je nach Fragestellung kann es jedoch auch einen kontinuierlichen Übergang zur Grundlagenforschung geben. Die Ressortforschung generiert idealtypisch Transferwissen und erbringt Übersetzungsleistungen vom wissenschaftlichen System in das Anwendersystem (z. B. Vollzug) und umgekehrt. Dabei verbindet sie kurzfristig abrufbare wissenschaftliche Kompetenz mit der Fähigkeit, langfristig angelegte Fragestellungen kontinuierlich bearbeiten zu können. Ressortforschung ist in der Regel in Behördenform institutionalisiert und unterliegt damit organisatorisch einer hierarchischen Organisationsstruktur. Die Ressortforschung des Bundes wird entweder durch die Bundesverwaltung selbst durchgeführt oder aber mittels Forschungsverträgen vorgenommen. In der Praxis geschieht dies meist über eigene nachgeordnete wissenschaftliche Fachbehörden und durch die Vergabe von Forschungsaufträgen, z. B. an Universitäten, Forschungsinstitute wie der Leibnitz-Gemeinschaft oder an die Fraunhofer-Gesellschaft. Die hierarchische Organisationsstruktur der Ressortforschung hat den Vorteil, dass die Bundesregierung jederzeit zur Erledigung ihrer Verwaltungsarbeit oder auch zur gezielten Bewältigung komplexer politischer Situationen oder Krisen auf unabhängige, fundierte wissenschaftliche Kenntnissen zurückgreifen kann.

Ressortforschung ist in der Regel interdisziplinäre Forschung, die für die Wahrnehmung der Aufgaben eines modernen Staates eine wesentliche Funktion zu übernehmen hat. Sie gibt die Fragen der Gesellschaft weiter und liefert mit ihrem Instrumentarium Versuchsergebnisse, Modelle und Beobachtungen, welche die Konzeption von politischen Strategien ermöglichen, die auf offensichtlichen Tatsachen beruhen (evidence-based public policies). Erste Voraussetzung für eine erfolgreiche Ressortforschung ist also strategisches Denken, um jene Bereiche zu identifizieren, in denen die Forschung in politisch vertretbaren Fristen Antworten auf gesellschaftliche Fragestellungen zu finden vermag. Ressortinstitute haben eine „Antennenfunktion“, sie müssen frühzeitig erkennen, ob Risiken zunehmen, ob Handlungsbedarf besteht und ob die Politik informiert werden muss. In ihrer strategischen Funktion trägt die Ressortforschung im Idealfall auch zur Festlegung der politischen Ziele der Bundesregierung bei, die in der Folge in entsprechenden Gesetzesvorhaben oder Verordnungen zum Ausdruck kommen. Zweite Voraussetzung ist ein professionelles Forschungsmanagement, das Maßnahmen zur Wissensverbreitung und den Wissenstransfer hin zur Praxis einschließt. Dritte Voraussetzung schließlich ist die Schaffung von Rah- 
menbedingungen, welche die wissenschaftliche Exzellenz der Ressortforschung sichern.

\section{Die Geschichte der Ressortforschung in der Bundesrepublik Deutschland}

Die wachsende Abhängigkeit moderner Gesellschaften von Technik und Wissenschaft sowie die Betroffenheit von den direkten und indirekten Technik- und Technisierungsfolgen stellen eine enorme Herausforderung für Gesellschaft und damit auch für die Regierung dar (Grunwald, 2010). Dabei steigt der Beratungsbedarf von Entscheidungsträgern in der Politik immer weiter an. Historisch gesehen hat die systematische Einbindung von Wissenschaft und Forschung in den politischen Entscheidungs- und Gesetzgebungsprozessen in Deutschland Tradition. Sie hat ihren Anfang genommen in der technischen und wissenschaftlichen Entwicklung in der zweiten Hälfte des 19. Jahrhunderts. Hier hat sich ein Problemgemenge im Zuge der rasanten Industrialisierung herausgebildet, welches staatliches Handeln und Regeln z.B. gerade auch in sozial- und arbeitshygienischen Feldern dringend erforderlich machte. Der Staat hat deshalb Ressortforschungseinrichtungen geschaffen, um die systematische Einbindung von Knowhow aus Wissenschaft und Forschung in politische Entscheidungsprozesse sicherstellen zu können. Die Ressortforschung und die daraus resultierende wissenschaftliche Politikberatung bieten Entscheidungsträgern in der Politik den Vorteil, direkt, kontinuierlich und zuverlässig unmittelbar abrufbares Expertenwissen vorzuhalten. Anhand des Praxisbeispieles der Ressortforschungsaktivitäten der Bundesanstalt für Arbeitschutz und Arbeitsmedizin (BAuA) sollen die typischen Handlungsweisen einer Ressortforschungsanstalt aufgezeigt werden.

\section{Strukturen, Prozesse und Arbeitsweise der Politikberatung in der BAuA}

Eine der wichtigsten strategischen Zukunftsaufgaben einer Ressortforschungseinrichtung ist die wissenschaftliche Politikberatung basierend auf eigener Forschungs- und Entwicklungsarbeit. Die Bundesanstalt für Arbeitsschutz und Arbeitsmedizin (BAuA) ist die einzige Ressortforschungseinrichtung im Geschäftsbereich des Bundesministeriums für Arbeit und Soziales (BMAS). Rund 660 Beschäftigte arbeiten am Hauptsitz in Dortmund und den Standorten Berlin, Dresden sowie in der Außenstelle Chemnitz.

Die Aufgabe der Bundesanstalt für Arbeitsschutz und Arbeitsmedizin ist es, für sichere und gesunde Arbeitsbedingungen Sorge zu tragen und dem BMAS fachliche Expertise und übergreifende Beratungsleistungen zur Verfügung zu stellen. Als Ressortforschungseinrichtung des Bundes zeigt die Bundesanstalt langfristig gültige Trends bei Sicherheit und Gesundheit am Arbeitsplatz auf und berät das Ministerium bei der Gesetzgebung: Die Bundesanstalt forscht und entwickelt im Themenfeld Sicherheit und Gesundheit bei der Arbeit, fördert den Wissenstransfer in die Praxis, berät die Politik und erfüllt hoheitliche Aufgaben - im Gefahrstoffrecht, bei der
Produktsicherheit und mit dem Gesundheitsdatenarchiv. Guter Arbeitsschutz bedeutet letztendlich sozialer Fortschritt und gibt den Unternehmen wie auch der gesamten Volkswirtschaft einen Vorsprung im globalen Wettbewerb.

Die BAuA berät die Politik in Ausschüssen und Gremien und beantwortet Anfragen der Bundesregierung. Zu den zentralen Aufgaben der fachpolitischen Politikberatung gehört insbesondere die Geschäftsführung und inhaltliche Ausgestaltung zentraler politikberatender Gremien, wie zum Beispiel:

v des Ausschusses für Arbeitsmedizin (AfaMed)

- des Ausschusses für Arbeitsstätten (ASTA)

- des Ausschusses für Betriebssicherheit (ABS)

- des Ausschusses für Biologische Arbeitsstoffe (ABAS)

- des Ausschusses für Gefahrenstoffe (AGS)

- des Ausschusses für technische Arbeitsmittel und Verbraucherprodukte (AtAV)

- EG-Gremien und EG-Arbeitsgruppen zur Erarbeitung und Durchführung von EG-Verordnungen und EG-Richtlinien

- Beraterkreise und Referentenbesprechungen des BMAS zu nationalen Vorschriften

- Ausschüsse der Bundesregierung nach Gesetzen, Rechtsverordnungen und Ministererlassen.

Bei den Ausschüssen ist das wesentliche Ziel, den Transfer des Expertenwissens der BAuA in die Praxis zu ermöglichen und eine Vereinheitlichung der Arbeitsweisen in den Ausschüssen und Maßnahmen der Qualitätssicherung des technischen Regelwerkes zu erreichen. Die wissenschaftliche Politikberatung zu fachpolitischen Fragen erfolgt in der Regel mittels Expertisen, Stellungnahmen, Konzeptpapieren, Berichten und Analysen. Dazu gehören auch die Fortentwicklung der EU-Richtlinien, der nationalen Vorschriften sowie die Normung. Darüber hinaus ist es das Ziel der BAuA, zukünftige Handlungsfelder im Bereich des Arbeitsschutzes wie zum Beispiel psychische Belastungen, Zeitarbeit oder Beschäftigungsfähigkeit zu identifizieren und hier ihr Expertenwissen proaktiv dem Ministerium für Arbeit und Soziales zur Verfügung zu stellen.

\section{Herausforderungen}

Die wissenschaftliche Politikberatung in einer Ressortforschungsanstalt ist im Idealfall eine Gemeinschaftsaufgabe von Wissenschaft und Politik. Deshalb ist für eine erfolgreiche Politikberatung wichtig, die Unterschiede zwischen den Zielsystemen der Wissenschaft und der Politik zu verstehen: Während die Wissenschaft beispielsweise oft sehr langfristig denkt und sich mit konkreten politischen Empfehlungen eher zurückhält, kommt es in der Politik auf oftmals sehr schnelle Entscheidungen an und auf die Berücksichtigung von politischen Opportunitäten. Ein weiterer wichtiger Punkt ist das Vertrauen: Wie auch die derzeitige EHEC-Krise zeigt, ist es wichtig, dass aufseiten der Politik und der Öffentlichkeit Vertrauen in die Ressortforschung vorhanden ist. Die Wissenschaft muss sich durch Kompetenz, Anerkennung und Kom- 
munikationsfähigkeit qualifizieren, politische Neutralität und persönliche Integrität müssen hinzutreten. Aus diesen Anforderungen lassen sich Kriterien für die Qualitätssicherung der Politikberatung ableiten. Um die Leistungsfähigkeit der Ressortforschungseinrichtungen $\mathrm{zu}$ sichern, sind qualitätssichernde Maßnahmen auf mehreren Ebenen notwendig. Hinweise zur Qualität der Politikberatung ergeben sich z. B. aus dem guten Ruf einer Einrichtung und ihrer profilierten Vertreter. Forschungserfolge sind ein indirektes Qualitätskriterium. Der Einfluss der Beratungstätigkeit auf politische Entscheidungen kann ein direkter Beleg für Beratungsqualität sein, in der Praxis ist der Einfluss aber oftmals nur sehr mittelbar nachvollziehbar. Neben der Entwicklung von belastbaren Qualitätskriterien ist es aber ebenso unabdingbar, dauerhaft wissenschaftliche Expertise auf international wettbewerbsfähigem Niveau vorzuhalten. Dies erfordert eine eigenständige, qualifizierte und ausreichend finanzierte Forschung. Gute, eigenständige Forschungsleistungen sind die notwendige Grundlage für eine gute Politikberatung und für hochwertige Dienstleistungen.

Um die Zukunft der Ressortforschung in der Bundesrepublik zu sichern, hat die Bundesregierung 2004 den Wissenschaftsrat beauftragt, die Ressortforschungseinrichtungen zu evaluieren. Mit der Verabschiedung übergreifender Empfehlungen zur Profilierung der Einrichtungen mit Ressortforschungsaufgaben des Bundes hat der Wissenschaftsrat 2010 die Begutachtung der Bundesressortforschung abgeschlossen. Insgesamt lobte der Wissenschaftsrat die hohe Leistungsfähigkeit der Ressortforschungseinrichtungen, aber es wurden auch umfangreiche Empfehlungen an die Bundesregierung ausgesprochen. Ziel dieser Empfehlungen war es, die Leistungsfähigkeit der Ressortforschungseinrichtungen zur Unterstützung der Bundesregierung zu stärken. Insbesondere wurden Maßnahmen zur Sicherung ihrer wissenschaftlichen Qualität angeregt. Auch sollten Ressortforschungseinrichtungen enger mit Hochschulen und außeruniversitären Forschungseinrichtungen zusammenarbeiten. Als wichtig sah es der Wissenschaftsrat auch an, flexiblere Regelungen im Personal- und Haushaltswesen einzurichten. Insbesondere wurde die Einrichtung von befristeten Stellen für wissenschaftliche Nachwuchskräfte und die Einführung von Globalhaushalten gefordert. Der Wissenschaftsrat forderte auch, dass die forschungsstärkeren Ressortforschungseinrichtungen in die Initiative Wissenschaftsfreiheitsgesetz der Bundesregierung einzubeziehen seien.

Ebenfalls an die Bundesregierung richten sich die Empfehlungen zu einer transparenteren Gestaltung des gesamten Bereichs der Ressortforschung. In diesem Zusammenhang trat der Wissenschaftsrat vor allem für eine regelmäßige Überprüfung der Forschungsbedarfe ein. Auf dieser Grundlage sollte die Bundesregierung entscheiden, ob Ressortforschungseinrichtungen gegründet, umstrukturiert oder geschlossen werden. Unabhängig davon empfiehlt der Wissenschaftsrat der Bundesregierung, alle Bundesbehörden, die in nennenswertem Umfang Forschungstätigkeiten durchführen, im Bundesforschungsbe- richt auszuweisen. Dies ist bislang nicht der Fall, für die Sichtbarkeit der Ressortforschungseinrichtungen im Wissenschaftssystem jedoch unerlässlich.

Verbesserungsbedarf hat der Wissenschaftsrat schließlich auch im Hinblick auf die internationale Einbindung der Ressortforschungseinrichtungen festgestellt. Er empfiehlt, die internationalen Aktivitäten der Ressortforschungseinrichtungen ressortübergreifend besser zu koordinieren sowie gemeinsam insbesondere mit den europäischen Partnern Möglichkeiten der Zusammenarbeit und der Arbeitsteilung zu prüfen und zu nutzen. Die deutsche Ressortforschung muss sich auch international besser vernetzen - nicht nur, aber auch im wissenschaftlichen Bereich. Dies sollte sich auch in der Forschungsplanung, im Organisationsaufbau sowie in der Personal- und Haushaltsführung niederschlagen. Die umfassende Integration der Ressortforschung in die Strategie der Bundesregierung zur Internationalisierung von Wissenschaft und Forschung wäre aus Sicht des Wissenschaftsrates hierfür ein wichtiger erster Schritt.

\section{Literatur}

Arbeitsgemeinschaft Ressortforschungseinrichtungen (2004. Forschen prüfen - beraten. Ressortforschungseinrichtungen als Dienstleister für Politik und Gesellschaft. Positionspapier Oktober 2004.

Bundesministerium für Bildung und Forschung (BMBF) (2007). Konzept einer modernen Ressortforschung. Bonn.

Bundesministerium für Bildung und Forschung (BMBF) (2007). Zehn Leitlinien einer modernen Ressortforschung. Bonn.

Grunwald, A. (2010). Technikfolgenabschätzung - ein Einführung. Berlin: Edition sigma.

Wissenschaftsrat (2010). Empfehlungen zur Profilierung der Einrichtungen mit Ressortforschungsaufgaben des Bundes. (Drs. 10295-10).

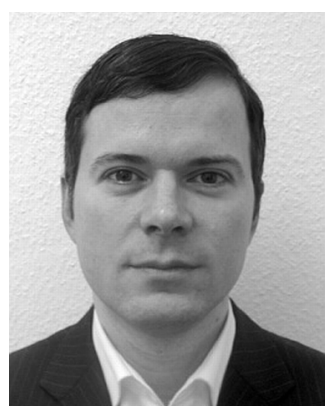

Andreas Süssmilch ist seit 2010 wissenschaftlicher Politikberater bei der Bundesanstalt für Arbeitsschutz und Arbeitsmedizin (BAuA). Von 2008-2010 war er Policy Advisor der Labour Party in London und zuvor als Policy Officer bei der Research Defence Society in London. Er studierte Politikwissenschaften und Geschichte in München, Berlin und London. E-Mail: Suessmilch.andreas@baua.bund.de 